\title{
Early life adversity is associated with a smaller hippocampus in male but not female depressed in-patients: a case- control study
}

\author{
Romain Colle ${ }^{1,2,3^{*}+}$, Tomoyuki Segawa ${ }^{1,2,3+}$, Marie Chupin ${ }^{4,5,6,7,8,9}$, Minh Ngoc Thien Kim Tran Dong ${ }^{10,11}$, \\ Patrick Hardy ${ }^{1,2,3}$, Bruno Falissard ${ }^{12}$, Olivier Colliot ${ }^{4,5,6,7,8,9}$, Denis Ducreux ${ }^{10,11}$ and Emmanuelle Corruble ${ }^{1,2,3}$
}

\begin{abstract}
Background: Three studies assessed the association of early life adversity (ELA) and hippocampal volumes in depressed patients, of which one was negative and the two others did not control for several potential confounding variables. Since the association of ELA and hippocampal volumes differ in male and female healthy volunteers, we investigated the association of ELA and hippocampal volumes in depressed patients, while focusing specifically on sex and controlling for several relevant socio-demographic and clinical variables.
\end{abstract}

Methods: Sixty-three depressed in-patients treated in a psychiatric setting, with a current Major Depressive Episode (MDE) and a Major Depressive Disorder (MDD) were included and assessed for ELA. Hippocampal volumes were measured with brain magnetic resonance imaging (MRI) and automatic segmentation. They were compared between patients with $(n=28)$ or without $(n=35)$ ELA. After bivariate analyses, multivariate regression analyses tested the interaction of sex and ELA on hippocampal volume and were adjusted for several potential confounding variables. The subgroups of men $(n=26)$ and women ( $n=37)$ were assessed separately.

Results: Patients with ELA had a smaller hippocampus than those without ELA $\left(4.65( \pm 1.11) \mathrm{cm}^{3}\right.$ versus 5.25 ( \pm 1.01$)$ $\mathrm{cm}^{3}$ ), bivariate: $p=0.03$, multivariate: $\left.H R=0.40,95 \% \mathrm{Cl}[0.23 ; 0.71], p=0.002\right)$, independently from other factors. This association was found in men $\left(4.43( \pm 1.22)\right.$ versus $\left.5.67( \pm 0.77) \mathrm{cm}^{3}\right)$, bivariate: $p=0.006$, multivariate $\mathrm{HR}=0.23,95 \% \mathrm{Cl}$ [0.06;0.82], $p=0.03)$ but not in women.

Conclusion: ELA is associated with a smaller hippocampus in male but not female depressed in-patients. The reasons for this association should be investigated in further studies.

Keywords: Early life adversity, Hippocampal volume, Sex, Major depressive disorder, Major depressive episode, MRI, Automated segmentation

\section{Background}

Early Life Adversity (ELA) has deleterious consequences during childhood and throughout life [1]. ELA is found in one out of two adult patients with Major Depressive Disorder (MDD) [2-7]. And adult patients with MDD

\footnotetext{
* Correspondence: romaincolle@hotmail.com

†Equal contributors

"INSERM UMRS 1178, Team "Depression and Antidepressants", 94275 Le Kremlin Bicêtre, France

${ }^{2}$ Faculté de Médecine Paris Sud, Univ. Paris-Sud, 94275 Le Kremlin Bicêtre, France

Full list of author information is available at the end of the article
}

have a two-fold higher rate of ELA compared to healthy individuals [8].

Interestingly, ELA may have a different impact on men and women. Indeed, epidemiological data show that ELA increases the risk for depression differently in men and women [9]. Moreover, the brain maturation [10] and reorganization of the inhibitory control network [11] after ELA may differ between men and women.

Recently, biomarker research in MDD [12] has focused on the hippocampus. In particular, hippocampal volume has been identified as a promising 
biomarker, smaller in MDD patients as compared to healthy subjects $[13,14]$. Moreover, functional Magnetic Resonance Imaging (fMRI) studies have shown changes in hippocampal activation associated with ELA (for review see Hart et al. 2012 [15]). In MDD, there are two studies showing differences in fMRI depending on the presence or absence of ELA. The first one in MDD children (20 boys and 22 girls) [16] reports that higher levels of ELA are associated with higher hippocampal activation in response to sad faces. The second one in 38 adult MDD patients reports that ELA is associated with a lower resting state connectivity in the limbic network, including the hippocampus [17]. In these two studies however, the impact of sex is not assessed.

Paradoxically, whereas the structural MRI biomarkers could be more easily transferable to clinical practice than functional MRI biomarkers, the literature linking ELA and hippocampal volumes in MDD is relatively poor, since only three studies are available $[5,18,19]$. The first study [18] performed in a sample of 31 women with remitted MDD fail to show an association between ELA and hippocampal volumes, even after controlling for age. In a second study of 37 MDD patients (16 men and 21 women) assessed with voxel based morphometry, Chaney et al. (2014) [5] report lower hippocampal grey matter in patients with ELA as compared to those without ELA, for the right but not for the left hippocampus. In a third study of 85 MDD patients (31 men and 54 women) assessed with a morphometric approach [19], Opel et al. (2014) show that ELA is associated with reduced hippocampal volumes. However, neither Chaney et al. (2014) nor Opel et al. (2014) controlled for sociodemographical or clinical variables, nor assessed specifically the role of sex. This point is crucial since hippocampal volumes are larger in men than in women $[20,21]$ and since a study in the general population shows that men with ELA have smaller hippocampal volumes than men without ELA whereas no difference is shown in women [22]. Regarding other relevant socio-demographic and clinical variables, older age [23], early age at onset of MDD [13], longer MDD duration [24], suicide attempts $[25,26]$ and smaller brain volume [20], are associated with smaller hippocampal volumes. Conversely, medication with antidepressants is associated with greater hippocampal volume [27]. And ELA is associated with early age at onset of MDD $[28,29]$ and higher depression severity [30].

Hence, the aim of our study was to investigate the association of ELA and hippocampal volumes in depressed patients, while focusing specifically on sex and controlling for several relevant socio-demographic and clinical variables.

\section{Methods}

\section{Design}

In this mono-centered study, the association between ELA and hippocampal volumes was assessed in adult depressed in-patients. This study was registered by the Commission Nationale de l'Informatique et des Libertés (CNIL) and was approved by the Ethics Committee of Paris-Boulogne, France, and conformed to international ethical standards and the latest version of the Declaration of Helsinki.

\section{Patients}

Consecutive in-patients, aged 18-65 years, with a diagnosis of a current Major Depressive Episode (MDE) in a context of MDD (DSM-IVTR) based on the Mini International Neuropsychiatric Interview (MINI) [31] and a Hamilton Depression Rating Scale 17 items (HDRS) [32] score of 18 or more, are included, prior to beginning a new antidepressant treatment. Patients with bipolar disorders, psychotic disorders, organic brain syndromes, unstable medical conditions, and contra-indications to cerebral MRI are not included. Alcohol dependence and marijuana use were associated with smaller hippocampal volume [33, 34] and also with ELA [35, 36] and MDD [37, 38]. Accordingly, patients with current substance abuse or dependence (DSM-IVTR) were not included in this study. In order to have a representative sample and increase the generalizability of our results, anxiety disorders and personality disorders, which are frequently comorbid with MDD and with ELA, are not excluded. Written informed consent of the participants is obtained after the nature of the procedures had been fully explained. All patients are hospitalized in the department of psychiatry of Bicêtre university hospital. They are systematically assessed by a psychiatrist for sex, age, age at onset of MDD, history of suicide attempt, depression severity with HDRS and lifetime medication with antidepressants. 68 patients were included but five patients were excluded from the analysis because of poor quality of hippocampal segmentations and/or MRI artefacts leading to an unreliable estimation of hippocampal volume. The sample analyzed comprises 63 patients. Their mean age is $46.4( \pm 12.4)$ years, $37(58.7 \%)$ are women, 7 (11.1\%) have a low educational level (French elementary school level, age: $2-11$ years), 31 (49.2\%) have a middle educational level (French secondary school level, age: 11-18 years), 25 (39.7\%) have a high educational level (French university level, age: 18 years and older), and 31 (49.2\%) are married. Regarding their MDD, 44 (69.8\%) have a recurrent MDD, the mean number of previous $\mathrm{MDE}$ is $2.4( \pm 1.6)$, the mean age at onset of MDD is $37.5( \pm 15.6)$ years, the mean MDD duration is 8.7 $( \pm 11.4)$ years, and $24(38.1 \%)$ patients are lifetime suicide attempters. Regarding antidepressants, 49 (78.8\%) were 
prescribed an antidepressant medication in the past and the mean duration of previous antidepressant treatment was $2.8( \pm 11.4)$ years. Thirty-four $(54.0 \%)$ patients received an antidepressant medication the week before assessment (selective serotonin reuptake inhibitors: $n=8$ $(23.5 \%)$, serotonin and norepinephrin reuptake inhibitors: $n=15$ (44.1\%), tricyclics: $n=5$ (14.7\%), others: $n=6$ $(17.6 \%))$, but this medication regimen was stopped two days before the assessment.

\section{Early life adversity}

ELA is defined here by either the death of caregivers or child abuse/maltreatment [1,39]. Child abuse/maltreatment was defined by all forms of physical and/or emotional ill-treatment, sexual abuse, neglect or negligent treatment or commercial or other exploitation, resulting in actual or potential harm to the child's health, survival, development or dignity in the context of a relationship of responsibility, trust or power [40].

Assessment of ELA was performed by 2 independent psychiatrists (TS and RC), using patient health records. TS and RC were not involved in the treatment of these patients and were blind to hippocampal volumes. Patient health records were documented during the hospitalization. Informations were obtained from the patients and close relationships, by several professionals, including senior psychiatrists, resident psychiatrists, general practitioners, other physicians who took care of this particular patient, nurses, psychologists and social workers. Indeed, during their hospitalization, patients benefited from multiple interviews with these professionals, who were blind from the objectives of the study. After the end of the hospitalization, each patient's health record was screened for the presence/absence of each component of ELA. ELA, i.e. death of caregiver, sexual abuse, emotional abuse, physical and emotional neglect, verbal abuse [39, 41, 42], was assessed as present if there was at least the death of a caregiver or one type of abuse (physical, verbal, sexual or emotional) or neglect (physical or emotional). Otherwise, ELA was assessed as absent. Afterwards, TS and RC reviewed the health records during a consensus group meeting supervised by EC, in order to obtain an agreement on the presence/absence of ELA.

\section{Brain magnetic resonance imaging}

Brain MRI acquisition methods were previously described [25]. Brain MRI acquisitions were performed on $1.5 \mathrm{~T}(n=47)$ or $3 \mathrm{~T}(n=16)$ Philips systems. All subjects were scanned with a routine whole brain T1weighted 3D sequence. These images were acquired with a resolution of either: sequence 1: $0.6 \times 0.6 \times 0.7$ (interpolated) in sagittal plane $(n=10)$; sequence 2: $0.94 \times$
$0.94 \times 1.00$ in axial plane $(n=37)$; sequence $3: 0.88 \times$ $0.88 \times 1.1$ in sagittal plane $(n=16)$. Since there were three different MRI acquisition sequences, the MRI acquisition method was added as a covariable in the multivariate analyses. The different acquisition sequences were not statistically different in men and women and in patients with or without ELA. The segmentation of the hippocampus was performed using the fully automatic SACHA software [43-46]. This approach segments the hippocampus based on competitive region-growing between the hippocampus and amygdala. It includes prior knowledge of the location of the hippocampus and the amygdala derived from a probabilistic atlas and on the relative positions of these structures with respect to anatomical landmarks, which are automatically identified. All resulting segmentations were assessed for segmentation quality (from 0 for worst quality to 4 for perfect quality) by trained raters (R.C and M.C), blind to the sociodemographic and clinical data. Only high quality segmentations (quality score $\geq 2$ ) were included in the analyses. Three variables of interest were studied on the basis of previous published papers [14, 47, 48]. Total hippocampal volume was the main assessment criterion. Right and left hippocampal volumes were also analyzed individually. Total brain volumes were estimated with SPM5 to control hippocampal volumes for this variable.

\section{Statistical methods}

ELA is the main independent variable and total hippocampal volume is the main dependent variable. After descriptive analyses, bivariate analyses were performed using Chi2 tests for categorical variables and Wilcoxon tests for continuous variables. After bivariate analyses, multivariate linear regressions were computed testing the interaction of sex and ELA on hippocampal volume and were adjusted on several variables, which could be confounders of the association between ELA and the hippocampal volumes. This possibility is based on data from the literature as well as results from bivariate analyses. Accordingly, the analyses were adjusted on age, brain volumes, MRI acquisition method, age at onset of MDD, history of suicide attempts, previous antidepressant medication and HDRS scores. Longer MDD duration was previously reported to be associated with smaller hippocampal volume [24]. However, since this variable was negatively correlated with age at onset of $\operatorname{MDD}(r=-0.6, p=0.000002$ in our sample), only age at onset of MDD was included in the multivariate analysis. Moreover, since the use of 3 different MRI acquisition sequences may bias our results, the multivariate analyses were adjusted on the MRI acquisition method (sequence 1 , sequence 2 and sequence 3 ).

In addition, the interaction between ELA and sex was specifically studied in the multivariate linear regressions. 
In the case of significant interactions, bivariate analyses were performed to test the association between ELA and hippocampal volumes in the subgroups of women and men.

All tests were two-tailed. Significance level was defined as $p<0.05$. The software used was $\mathrm{R} 3.2 .2$ (www.r-project.org).

\section{Results}

ELA is found in 28 (44.4\%) patients. Multiple ELA are found in 12 patients. The types of ELA are: death of caregiver $(11(17.4 \%))$, physical abuse $(8(12.7 \%))$, emotional abuse $(4(6.3 \%))$, sexual abuse $(2(3.2 \%))$, verbal abuse $(5(7.9 \%))$, physical neglect $(4(6.3 \%))$ and emotional neglect (17 (27.0\%)).

Patients with and without ELA do not differ in terms of sex and other socio-demographic variables and MDD features, except for the age at onset of MDD, which is earlier in patients with ELA as compared to those without ELA (Table 1).

Significant and independent associations are shown between ELA and hippocampal volumes (Table 2). As compared to patients without ELA, patients with ELA have smaller total hippocampal volumes (Fig. 1), as evidenced in the bivariate $(w=644.5, p=0.03)$ and multivariate $(H R=0.40, \quad 95 \% \mathrm{CI} \quad[0.23 ; 0.71], \quad p=0.002)$ analyses. They also had smaller right hippocampal volumes (bivariate: $w=570, p=0.10$; multivariate: $H R=$ 0.67 , CI95\% CI [0.49;0.93], $p=0.02$ ) and smaller left hippocampal volumes (bivariate: $w=574, p=0.06$; multivariate: $H R=0.66,95 \% \mathrm{CI}[0.48 ; 0.89], p=0.007)$.

Table 1 Socio-demographical and clinical data in patients with or without early life adversity

\begin{tabular}{lll}
\hline & ELA+ $(n=28)$ & ELA- $(n=35)$ \\
\hline Women $n(\%)$ & $19(67.9)$ & $18(51.4)$ \\
Age (years) (m (sd)) & $43.6(12.4)$ & $48.5(12.1)$ \\
Educational level (\%) & & \\
$\quad$ Low & $2(7.1)$ & $5(14.3)$ \\
Middle & $17(60.7)$ & $14(40.0)$ \\
High & $9(32.1)$ & $16(45.7)$ \\
Age at onset of MDD (m (sd)) a & $32.9(15.1)$ & $41.2(15.3)$ \\
MDD duration (m (sd)) & $10.7(12.5)$ & $7.1(10.3)$ \\
Recurrent MDD (\%) & $21(75.0)$ & $23(65.7)$ \\
Number of previous MDE (m (sd)) & $2.3(1.6)$ & $2.4(1.7)$ \\
HDRS (m (sd)) & $23.8(5.2)$ & $24.5(5.7)$ \\
Previous antidepressant medication (\%) & $22(78.6)$ & $27(77.1)$ \\
Suicide Attempts (\%) & $10(35.7)$ & $14(40.0)$ \\
\hline
\end{tabular}

ELA early life adversity, ELA+ presence of early life adversity, ELA- absence of early life adversity, $M D D$ major depressive disorder, $M D E$ major depressive episode, HDRS hamilton depression rating scale 17 items, ${ }^{a}: w=619, p=0.04$
In multivariate analyses (Table 2), significant and independent associations are also shown between three other variables (brain volume, MRI acquisition method and history of suicide attempts) and hippocampal volumes.

A significant interaction between ELA and sex is shown for total hippocampal volumes $(p=0.04)$ and left hippocampal volumes $(p=0.03)$ (Table 2).

In the subgroup of men $(n=26)$, those with ELA have smaller total, right and left hippocampal volumes than those without ELA (respectively $p=0.06, p=0.01$ and $p=0.02$ in bivariate analyses, Table 3 ). Multivariate analyses adjusted for age, brain volumes, MRI acquisition method, age at onset of MDD, history of suicide attempts, previous antidepressant medication and HDRS show that men with ELA have smaller total and right hippocampal volumes, but not left hippocampal volumes, than men without ELA (respectively $H R=0.23,95 \% \mathrm{CI}[0.06 ; 0.82], p=0.03 ; H R=$ $0.52,95 \% \mathrm{CI}[0.27 ; 1.00], p=0.05$; and $H R=0.72,95 \% \mathrm{CI}$ [0.23; 2.25], $p=0.55$ ).

In the subgroup of women $(n=37)$, no difference of hippocampal volumes (total, right or left) is shown between those with and without ELA (Table 3, Fig. 2).

\section{Discussion}

Our results show that ELA is associated with a smaller hippocampus in male but not female depressed in-patients treated in a psychiatric setting, even after controlling for several relevant socio-demographic and clinical variables. The main strengths of this study are that it focuses specifically on the effect of sex and that it controls for several relevant variables, such as age, age at onset of MDD, history of suicide attempts, previous antidepressant medication and Hamilton Depression Rating Scale scores.

This result is coherent with the result of Samplin et al. (2013) in healthy volunteers [22], which shows that healthy men with ELA have smaller hippocampal volumes than healthy men without ELA, while no difference was evidenced in women. It suggests that our result might not be specific to MDD patients. However, the effect could be more pronounced in depressed patients, since depressed men with ELA in our sample have a $21.9 \%$ lower hippocampal volume than depressed men without ELA, whereas the difference was $7.3 \%$ in the sample of male healthy volunteers of Samplin et al. (2013) [22]. Other studies concerning healthy subjects $[19,49-51]$ report smaller hippocampal volumes in individuals with ELA. However, the effect of gender was not specifically assessed in these studies. Our results are also coherent with those of Lenze et al. (2008) [18], who show no association between ELA and hippocampal volumes in a sample of 31 women with MDD. But our results go beyond those of Chaney et al. (2014) and Opel et al. (2014), who did not control for socio- 
Table 2 Results of multivariate models for total, right and left hippocampal volumes

\begin{tabular}{|c|c|c|c|c|c|c|}
\hline & \multicolumn{2}{|c|}{ Total Hippocampal Volume } & \multicolumn{2}{|c|}{ Right Hippocampal Volume } & \multicolumn{2}{|c|}{ Left Hippocampal Volume } \\
\hline & estimate & $p$ & estimate & $p$ & estimate & $p$ \\
\hline$E L A+(v s$ ELA-) & -0.91 & 0.002 & -0.40 & 0.02 & -0.43 & 0.007 \\
\hline Sex (women vs men) & 0.37 & 0.26 & 0.18 & 0.31 & 0.18 & 0.31 \\
\hline Age & 0.00 & 0.81 & 0.00 & 0.16 & 0.00 & 0.83 \\
\hline Brain volume & 0.00 & 0.07 & 0.00 & 0.52 & 0.00 & 0.004 \\
\hline \multicolumn{7}{|l|}{ MRI acquisition method } \\
\hline (sequence 2 vs 1) & 1.28 & 0.002 & 0.85 & 0.0003 & 0.37 & 0.08 \\
\hline (sequence 3 vs 1) & 1.31 & 0.003 & 0.77 & 0.002 & 0.59 & 0.01 \\
\hline MDD age at onset & -0.01 & 0.68 & -0.00 & 0.66 & -0.00 & 0.76 \\
\hline History of suicide attempts (yes vs no) & -0.66 & 0.02 & -0.37 & 0.01 & -0.17 & 0.25 \\
\hline Previous antidepressant medication (yes vs no) & -0.01 & 0.98 & -1.17 & 0.25 & 0.18 & 0.36 \\
\hline HDRS score & -0.01 & 0.61 & -0.01 & 0.58 & -0.01 & 0.49 \\
\hline ELA and Sex interaction & 1.16 & 0.03 & 0.52 & 0.10 & 0.61 & 0.03 \\
\hline
\end{tabular}

ELA early life adversity, ELA+ presence of early life adversity, ELA- absence of early life adversity, MRI acquisition: sequence $1: 0.6 \times 0.6 \times 0.7$ (interpolated) in sagittal plane, sequence 2: $0.94 \times 0.94 \times 1.00$ in axial plane; sequence 3: $0.88 \times 0.88 \times 1.1$ in sagittal plane, MDD major depressive disorder, $H D R S$ hamilton depression rating scale 17 items; bold: $p$ value $<0.05$

demographical or clinical variables, and did not assess specifically the role of sex. Indeed, our study is the first one showing a specific effect of sex on the association between ELA and hippocampal volumes in MDD, this association being evidenced only in men. Moreover, our results are in line with those of the two fMRI studies concerning MDD [16, 17], although these two studies did not assess the effect of sex.

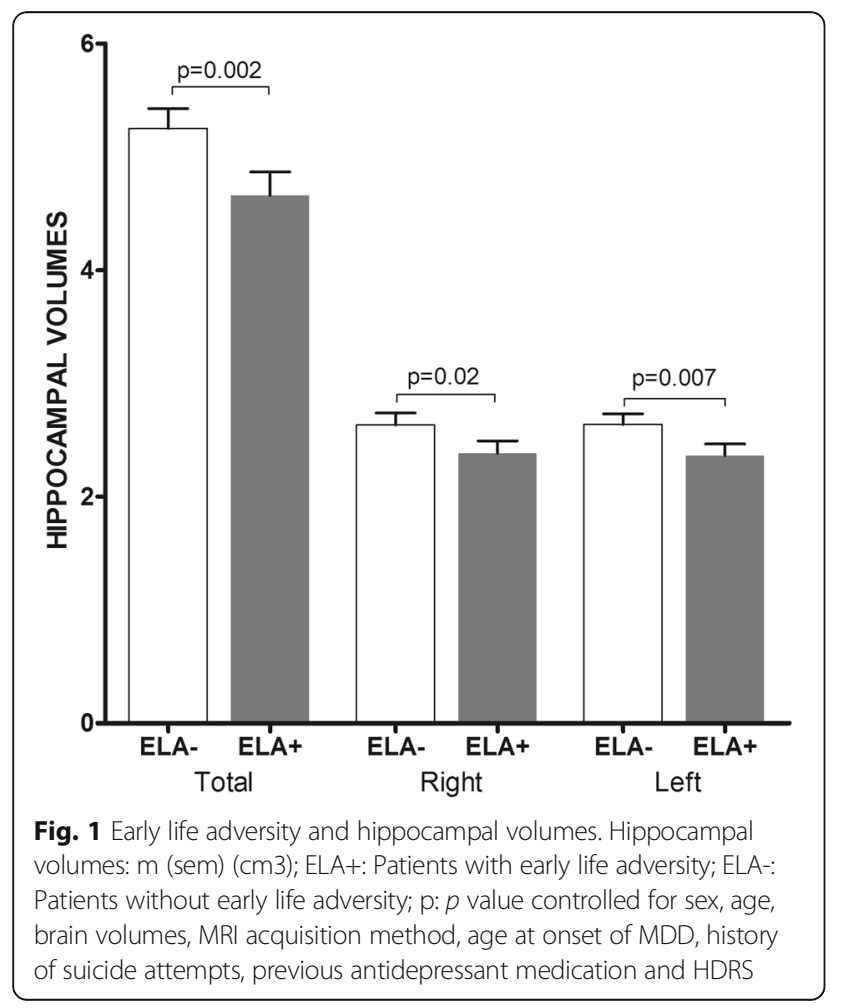

Moreover, our results are in line with those of a study in patients with chronic psychosis [52] although the specific effect of sex was not assessed. In addition, we show a specific and independent effect of the history of suicide attempts, which is coherent with previous results $[25,26]$. The non-inclusion of patients with current substance abuse or dependence is one of the strengths of this study. Thus alcohol consumption and marijuana use cannot bias our results.

It should be highlighted that several points argue for the generalizability of our results. They are in line with those of the literature for the frequency of ELA (44.4\% in our sample, 54\% in Chaney et al. (2014) [5], 51.4\% in Gerritsen et al. (2015) [6], for the magnitude of hippocampal volumes $[46,53]$ and for the association between ELA and earlier age at onset of MDD [28, 29].

Nevertheless, our study has some limitations. Firstly, ELA is retrospectively assessed as a dichotomous variable (absent/present), leading to possible memory biases among patients. However, we used a clinical assessment method, which could catch more relevant events than self-assessment methods. Our method is based on medical records, several information sources (i.e. patients and close relationships) and multiple interviews with multiple interviewers leading to possible higher sensitivity to detect ELA than a one-time self-assessment subjected to memory biases. However, our method is difficult to use with out-patients or healthy subjects, since it is time consuming, but it may be particularly relevant for in-patients. Nonetheless, our results should be replicated with validated assessment methods of ELA. This may include a clinician administered interview 
Table 3 Early life adversity and hippocampal volumes in men and women

\begin{tabular}{|c|c|c|c|c|c|c|}
\hline & \multicolumn{3}{|l|}{ Women } & \multicolumn{3}{|l|}{ Men } \\
\hline & $\mathrm{ELA}+(n=19)$ & $\mathrm{ELA}-(n=18)$ & p & $\mathrm{ELA}+(n=9)$ & $\mathrm{ELA}-(n=17)$ & $p$ \\
\hline Total hippocampal volume $\left(\mathrm{cm}^{3}\right)(\mathrm{m}(\mathrm{sd}))$ & $4.77(1.07)$ & $4.85(1.07)$ & 0.99 & $4.43(1.22)$ & $5.67(0.77)$ & 0.006 \\
\hline Right hippocampal volume $\left(\mathrm{cm}^{3}\right)(\mathrm{m}(\mathrm{sd}))$ & $2.43(0.59)$ & $2.45(0.64)$ & 0.99 & $2.28(0.57)$ & $2.82(0.49)$ & 0.01 \\
\hline Left hippocampal Volume $\left(\mathrm{cm}^{3}\right)(\mathrm{m}(\mathrm{sd}))$ & $2.41(0.50)$ & $2.43(0.51)$ & 0.98 & $2.23(0.66)$ & $2.86(0.45)$ & 0.02 \\
\hline
\end{tabular}

ELA early life adversity, ELA+ presence of early life adversity, ELA - absence of early life adversity, $p p$ values in bivariate analyses, bold: $p$ value $<0.05$

Childhood Experience of Care and Abuse (CECA) This may include a clinician administered interview of the Childhood Experience of Care and Abuse (CECA), or its self-report version, the CECA questionnaire, which assess several dimensions such as lack of parental care (neglect and antipathy), parental physical abuse, and sexual abuse from any adult before age 17 [54], or the Childhood Trauma Questionnaire [41] which is also a self-report method and the most used method assessing emotional and physical neglect and emotional, physical, and sexual abuse. However, standardized methods in depressed patients lead to frequencies of ELA which are similar to our results $(44.4 \%$ in our sample, $54 \%$ in Chaney et al. (2014) [5] with the Childhood Trauma

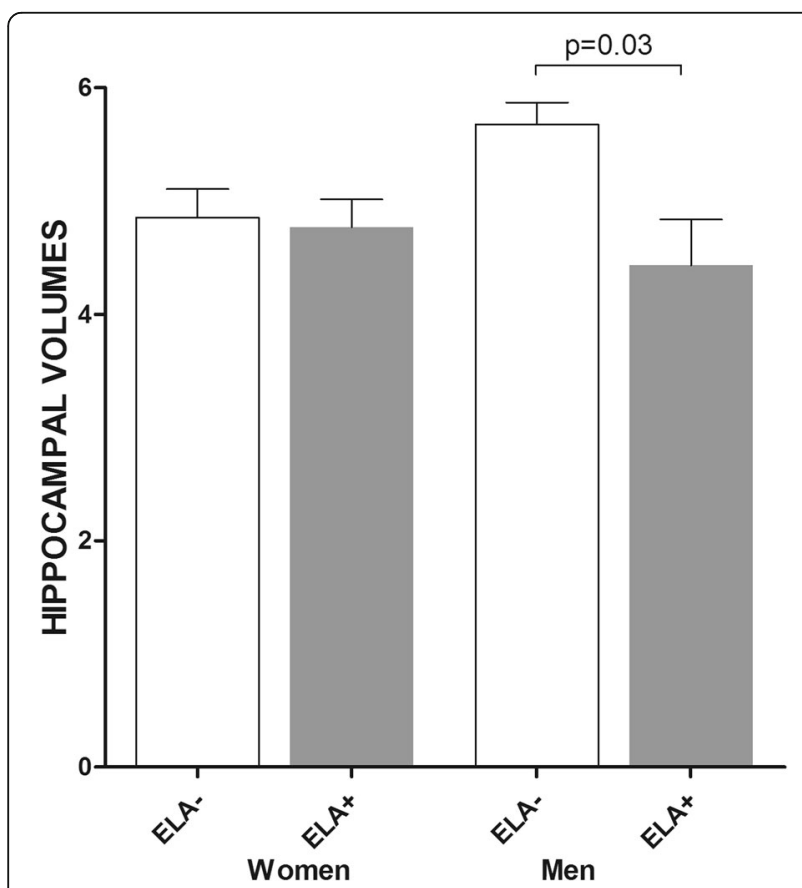

Fig. 2 Early life adversity and total hippocampal volumes in men and women. Hippocampal volumes: $m$ (sem) (cm3); ELA+: Patients with early life adversity; ELA-: Patients without early life adversity; p: $p$ value adjusted for age, brain volumes, MRI acquisition method, age at onset of MDD, history of suicide attempts, previous antidepressant medication and HDRS score
Questionnaire [41], 51.4\% in Gerritsen et al. (2015) [6] and with the Nemesis Trauma Interview [55].

Secondly, due to the small sample size, especially in the subgroup of men, we cannot exclude that our results may be false positives. However, their coherence with those shown in healthy volunteers [22] argue for their validity.

Thirdly, the power of the study was not sufficient to analyze the impact of specific types of ELA. Fourthly, the impact of anxiety disorders or personality disorders was not analyzed because these variables were not specifically assessed here.

Fifthly, this sample comprises only MDD in-patients treated in a psychiatric setting. Thus, our results cannot be generalized to the whole population of MDD patients. And, lastly, these results cannot be interpreted in terms of causality.

Nonetheless, the explanation for this association is unclear. However, it could be suggested that there is a mediating role of the dysregulation of the hypothalamic pituitary adrenal (HPA) axis Indeed, ELA is associated with a dysregulation of the HPA axis (for review see [56] and Struber et al. 2014 [57]). In addition, cortisol can have detrimental effects on hippocampal neurons [58]. Interestingly, a sex-dependent impact of ELA on saliva daily cortisol levels has been reported. Boys with ELA have a higher daily cortisol level than girls with ELA [59]. This higher cortisol level may explain the lower hippocampal volume shown in men but not in women with major depression. However, further studies are needed to test this hypothesis.

If they could be replicated in further studies, our results may have several implications. Generally speaking, it would be useful to pay more attention to ELA in boys and men, their assessment and potential impact on hippocampal volumes and MDD. More specifically, the benefits of psychotherapy in boys and men with ELA should be assessed to a greater degree. Finally, since men receive less appropriate mental health care than women for MDD [60], it would be useful to improve care access, specifically for men in order to treat MDD earlier in men. Finally, our results and their potential explanations should be studied further in longitudinal studies assessing ELA in children, and especially boys, and following 
them prospectively for both hippocampal volumes and MDD.

\section{Conclusion}

Early life adversity is associated with a smaller hippocampus in male but not female depressed in-patients, even after controlling for several potential confounders. The reasons for this association should be investigated in further studies.

\section{Abbreviations \\ CNIL: Commission Nationale de I'Informatique et des Libertés (CNIL); ELA: Early Life Adversity; HDRS: Hamilton Depression Rating Scale: MDD: Major Depressive Disorder; MDE: Major Depressive Episode; MRI: Magnetic Resonance Imaging}

\section{Acknowledgements}

Linguistic revision: Magda Rosinska

\section{Funding}

This study was funded by the Programme Hospitalier de Recherche Clinique from the French Ministry of Health (AOR10071) and the Assistance Publique Hôpitaux de Paris (P060219).

The funding source was not involved in the scientific process leading to the presentation of this paper.

\section{Availability of data and materia}

The dataset analyzed in this study is available from the corresponding author on reasonable request.

\section{Authors' contributions}

The authors' contributions are the following: RC: conception of the work, data collection, data management, analysis, drafting, interpretation of data, final approval. TS: data collection, drafting, interpretation of data, final approval. MC: volumetry assessment, interpretation of data, revising, final approval. MNTKTD: interpretation of data, revising, final approval. PH: clinica data acquisition, revising, final approval. BF: analysis, interpretation of data, revising, editing assistance, final approval. OC: volumetry assessment, interpretation of data, revising, final approval. DD: MRI acquisition, data collection, revising, final approval. EC: conception of the work, drafting, interpretation of data, final approval. RC and EC had full access to all the data of the study and take responsibility for the integrity of the data and the accuracy of the data analysis.

\section{Competing interests}

RC, TS, MC, MNTKTD, PH, DD have no conflicts of interest. BF has been consultant, expert or has given talks for E. Lilly, BMS, Servier, Sanofi, GlaxoSmithKline, HRA, Roche, Boeringer Ingelheim, Bayer, Almirall, Allergan, Stallergene, Genzyme, Pierre Fabre, Astra Zeneca, Novartis, Janssen, Astellas, Biotronik, Daiichi-Sankyo, Gilead, MSD, Lundbeck. OC received lecture fees from Lundbeck and consulting fees from Guerbet. EC received consulting or conference fees from Eisai, Lundbeck, Servier.

\section{Consent for publication}

Not applicable.

\section{Ethics approval and consent to participate}

This study was registered by the Commission Nationale de l'Informatique et des Libertés (CNIL) and was approved by the Ethics Committee of Paris-Boulogne, France, and conformed to international ethical standards and the latest version of the Declaration of Helsinki. Written informed consent of the participants was obtained after the nature of the procedures had been fully explained.

\section{Author details}

'INSERM UMRS 1178, Team "Depression and Antidepressants", 94275 Le Kremlin Bicêtre, France. 'Faculté de Médecine Paris Sud, Univ. Paris-Sud, 94275 Le Kremlin Bicêtre, France. ${ }^{3}$ Service de Psychiatrie, Hôpital Bicêtre, Hôpitaux Universitaires Paris Sud, Assistance Publique-Hôpitaux de Paris, 94275 Le Kremlin Bicêtre, France. ${ }^{4}$ INSERM U1127, F-75013 Paris, France.
${ }^{5}$ CNRS, UMR 7225, 75013 Paris, France. ${ }^{6}$ Sorbonne Universités, UPMC Univ. Paris 06, UMR S 1127, F-75013 Paris, France. Institut du Cerveau et de la Moelle épinière, ICM, F-75013 Paris, France. ${ }^{8}$ Inria, Aramis project-team, Centre de Recherche de Paris, Paris, France. ${ }^{9}$ Departments of Neuroradiology and Neurology, AP-HP, Hôpital de la Pitié-Salpêtrière, F-75013 Paris, France. ${ }^{10}$ CNRS IR4M, UMR 8081, 94275 Le Kremlin Bicêtre, France. ${ }^{11}$ Service de Neuroradiologie, Hôpital Bicêtre, Hôpitaux Universitaires Paris Sud, Assistance Publique Hôpitaux de Paris, 94275 Le Kremlin Bicêtre, France. ${ }^{12}$ INSERM, Université Paris-Saclay, Univ. Paris-Sud, UVSQ, CESP, Villejuif, France.

Received: 25 October 2016 Accepted: 7 February 2017

Published online: 15 February 2017

\section{References}

1. Cicchetti D, Doyle C. Child maltreatment, attachment and psychopathology: mediating relations. World Psychiatry. 2016;15(2):89-90.

2. Li M, D'Arcy C, Meng X. Maltreatment in childhood substantially increases the risk of adult depression and anxiety in prospective cohort studies: systematic review, meta-analysis, and proportional attributable fractions. Psychol Med. 2016:46(4):717-30.

3. Chapman DP, Whitfield CL, Felitti VJ, Dube SR, Edwards VJ, Anda RF. Adverse childhood experiences and the risk of depressive disorders in adulthood. J Affect Disord. 2004;82(2):217-25.

4. Nanni $V$, Uher $R$, Danese A. Childhood maltreatment predicts unfavorable course of illness and treatment outcome in depression: a meta-analysis. Am J Psychiatry. 2012;169(2):141-51.

5. Chaney A, Carballedo A, Amico F, Fagan A, Skokauskas N, Meaney J, et al. Effect of childhood maltreatment on brain structure in adult patients with major depressive disorder and healthy participants. J Psychiatry Neurosci. 2014;39(1):50-9.

6. Gerritsen L, Van Velzen L, Schmaal L, van der Graaf $Y$, van der Wee N, Van Tol MJ, et al. Childhood maltreatment modifies the relationship of depression with hippocampal volume. Psychol Med. 2015;45(16):3517-26.

7. Lindert J, Von Ehrenstein OS, Grashow R, Gal G, Braehler E, Weisskopf MG. Sexual and physical abuse in childhood is associated with depression and anxiety over the life course: systematic review and meta-analysis. Int $J$ Public Health. 2014;59(2):359-72.

8. Williams LM, Debattista C, Duchemin AM, Schatzberg AF, Nemeroff CB. Childhood trauma predicts antidepressant response in adults with major depression: data from the randomized international study to predict optimized treatment for depression. Transl Psychiatry. 2016;6:e799.

9. McCutcheon W, Heath AC, Nelson EC, Bucholz KK, Madden PA, Martin NG. Accumulation of trauma over time and risk for depression in a twin sample. Psychol Med. 2009;39(3):431-41.

10. De Bellis MD, Keshavan MS. Sex differences in brain maturation in maltreatment-related pediatric posttraumatic stress disorder. Neurosci Biobehav Rev. 2003:27(1-2):103-17.

11. Elton A, Tripathi SP, Mletzko T, Young J, Cisler JM, James GA, et al. Childhood maltreatment is associated with a sex-dependent functional reorganization of a brain inhibitory control network. Hum Brain Mapp. 2014;35(4):1654-67.

12. Kupfer DJ, Frank E, Phillips ML. Major depressive disorder: new clinical, neurobiological, and treatment perspectives. Lancet. 2012;379(9820):1045-55.

13. Schmaal L, Veltman DJ, Van Erp TG, Samann PG, Frodl T, Jahanshad N, et al. Subcortical brain alterations in major depressive disorder: findings from the ENIGMA Major Depressive Disorder working group. Mol Psychiatry. 2016:21(6):806-12.

14. Kempton MJ, Salvador Z, Munafo MR, Geddes JR, Simmons A, Frangou S, et al. Structural neuroimaging studies in major depressive disorder. Metaanalysis and comparison with bipolar disorder. Arch Gen Psychiatry. 2011;68(7):675-90.

15. Hart H, Rubia K. Neuroimaging of child abuse: a critical review. Front Hum Neurosci. 2012;6:52.

16. Suzuki H, Luby JL, Botteron KN, Dietrich R, McAvoy MP, Barch DM. Early life stress and trauma and enhanced limbic activation to emotionally valenced faces in depressed and healthy children. J Am Acad Child Adolesc Psychiatry. 2014;53(7):800-13. e810.

17. Wang L, Dai Z, Peng H, Tan L, Ding Y, He Z, et al. Overlapping and segregated resting-state functional connectivity in patients with major depressive disorder with and without childhood neglect. Hum Brain Mapp. 2014;35(4):1154-66. 
18. Lenze SN, Xiong C, Sheline YI. Childhood adversity predicts earlier onset of major depression but not reduced hippocampal volume. Psychiatry Res. 2008;162(1):39-49.

19. Opel N, Redlich R, Zwanzger P, Grotegerd D, Arolt V, Heindel W, et al. Hippocampal atrophy in major depression: a function of childhood maltreatment rather than diagnosis? Neuropsychopharmacology. 2014;39(12):2723-31.

20. Brown ES, Hughes CW, McColl R, Peshock R, King KS, Rush AJ. Association of depressive symptoms with hippocampal volume in 1936 adults. Neuropsychopharmacology. 2014;39(3):770-9.

21. Tan A, Ma W, Vira A, Marwha D, Eliot $L$. The human hippocampus is not sexuallydimorphic: Meta-analysis of structural MRI volumes. Neuroimage. 2016;124:350-66.

22. Samplin E, Ikuta T, Malhotra AK, Szeszko PR, Derosse P. Sex differences in resilience to childhood maltreatment: effects of trauma history on hippocampal volume, general cognition and subclinical psychosis in healthy adults. J Psychiatr Res. 2013:47(9):1174-9.

23. Daugherty AM, Bender AR, Raz N, Ofen N. Age differences in hippocampal subfield volumes from childhood to late adulthood. Hippocampus. 2016;26(2):220-8.

24. McKinnon MC, Yucel K, Nazarov A, MacQueen GM. A meta-analysis examining clinical predictors of hippocampal volume in patients with major depressive disorder. J Psychiatry Neurosci. 2009;34(1):41-54.

25. Colle R, Chupin M, Cury C, Vandendrie C, Gressier F, Hardy P, et al. Depressed suicide attempters have smaller hippocampus than depressed patients without suicide attempts. J Psychiatr Res. 2015;61:13-8.

26. Gosnell SN, Velasquez KM, Molfese DL, Molfese PJ, Madan A, Fowler JC, et al. Prefrontal cortex, temporal cortex, and hippocampus volume are affected in suicidal psychiatric patients. Psychiatry Res. 2016;256:50-6.

27. Arnone D, McKie S, Elliott R, Juhasz G, Thomas EJ, Downey D, et al. Statedependent changes in hippocampal grey matter in depression. Mol Psychiatry. 2013;18(12):1265-72.

28. Bernet CZ, Stein MB. Relationship of childhood maltreatment to the onset and course of major depression in adulthood. Depress Anxiety. 1999;9(4):169-74.

29. Tunnard C, Rane LJ, Wooderson SC, Markopoulou K, Poon L, Fekadu A, et al. The impact of childhood adversity on suicidality and clinical course in treatment-resistant depression. J Affect Disord. 2014;152-154:122-30.

30. Vogt D, Waeldin S, Hellhammer D, Meinlschmidt G. The role of early adversity and recent life stress in depression severity in an outpatient sample. J Psychiatr Res. 2016;83:61-70.

31. Sheehan DV, Lecrubier $Y$, Sheehan $\mathrm{KH}$, Amorim $\mathrm{P}$, Janavs J, Weiller $E$, et al. The Mini-International Neuropsychiatric Interview (M.I.N.I.): the development and validation of a structured diagnostic psychiatric interview for DSM-IV and ICD-10. J Clin Psychiatry. 1998;59(22-33):3-57.

32. Hamilton M. A rating scale for depression. J Neurol Neurosurg Psychiatry. 1960;23:56-62.

33. Kuhn $S$, Charlet $K$, Schubert F, Kiefer F, Zimmermann $P$, Heinz A, et al. Plasticity of hippocampal subfield volume cornu ammonis $2+3$ over the course of withdrawal in patients with alcohol dependence. JAMA Psychiatry. 2014;71(7):806-11.

34. Filbey FM, McQueeny T, Kadamangudi S, Bice C, Ketcherside A. Combined effects of marijuana and nicotine on memory performance and hippocampal volume. Behav Brain Res. 2015:293:46-53.

35. Enoch MA. The role of early life stress as a predictor for alcohol and drug dependence. Psychopharmacology (Berl). 2011;214(1):17-31.

36. Mills R, Kisely S, Alati R, Strathearn L, Najman JM. Child maltreatment and cannabis use in young adulthood: a birth cohort study. Addiction. 2016.

37. Boden JM, Fergusson DM. Alcohol and depression. Addiction. 2011;106(5):906-14.

38. Chen CY, Wagner FA, Anthony JC. Marijuana use and the risk of Major Depressive Episode. Epidemiological evidence from the United States National Comorbidity Survey. Soc Psychiatry Psychiatr Epidemiol. 2002;37(5):199-206.

39. Finkelstein $\mathrm{H}$. The long-term effects of early parent death: a review. J Clin Psychol. 1988;44(1):3-9.

40. Report of the Consultation on Child Abuse Prevention, 29-31 March 1999. [http://www.who.int/iris/handle/10665/65900].

41. Bernstein DP, Fink L, Handelsman L, Foote J, Lovejoy M, Wenzel K, et al. Initial reliability and validity of a new retrospective measure of child abuse and neglect. Am J Psychiatry. 1994;151(8):1132-6.

42. Ney PG. Does verbal abuse leave deeper scars: a study of children and parents. Can J Psychiatry. 1987;32(5):371-8

43. Chupin M, Hammers A, Bardinet E, Colliot O, Liu RS, Duncan JS, et al. Fully automatic segmentation of the hippocampus and the amygdala from MRI using hybrid prior knowledge. Med Image Comput Comput Assist Interv. 2007;10(Pt 1):875-82
44. Chupin M, Hammers A, Liu RS, Colliot O, Burdett J, Bardinet E, et al. Automatic segmentation of the hippocampus and the amygdala driven by hybrid constraints: method and validation. Neuroimage. 2009;46(3):749-61.

45. Colliot O, Chetelat G, Chupin M, Desgranges B, Magnin B, Benali H, et al. Discrimination between Alzheimer disease, mild cognitive impairment, and normal aging by using automated segmentation of the hippocampus. Radiology. 2008;248(1):194-201.

46. Bergouignan L, Chupin M, Czechowska Y, Kinkingnehun S, Lemogne C, Le Bastard G, et al. Can voxel based morphometry, manual segmentation and automated segmentation equally detect hippocampal volume differences in acute depression? Neuroimage. 2009:45(1):29-37.

47. Kronmuller KT, Pantel J, Kohler S, Victor D, Giesel F, Magnotta VA, et al. Hippocampal volume and 2-year outcome in depression. Br J Psychiatry. 2008;192(6):472-3.

48. Sheline YI, Disabato BM, Hranilovich J, Morris C, D'Angelo G, Pieper C, et al. Treatment course with antidepressant therapy in late-life depression. Am J Psychiatry. 2012;169(11):1185-93.

49. Dannlowski U, Stuhrmann A, Beutelmann V, Zwanzger P, Lenzen T, Grotegerd D, et al. Limbic scars: long-term consequences of childhood maltreatment revealed by functional and structural magnetic resonance imaging. Biol Psychiatry. 2012;71(4):286-93.

50. Gorka AX, Hanson JL, Radtke SR, Hariri AR. Reduced hippocampal and medial prefrontal gray matter mediate the association between reported childhood maltreatment and trait anxiety in adulthood and predict sensitivity to future life stress. Biol Mood Anxiety Disord. 2014;4:12.

51. Teicher $\mathrm{MH}$, Anderson $\mathrm{CM}$, Polcari $\mathrm{A}$. Childhood maltreatment is associated with reduced volume in the hippocampal subfields CA3, dentate gyrus, and subiculum. Proc Natl Acad Sci U S A. 2012;109(9):E563-72.

52. Hoy K, Barrett S, Shannon C, Campbell C, Watson D, Rushe T, et al. Childhood trauma and hippocampal and amygdalar volumes in firstepisode psychosis. Schizophr Bull. 2012;38(6):1162-9.

53. MacQueen GM, Yucel K, Taylor VH, Macdonald K, Joffe R. Posterior hippocampal volumes are associated with remission rates in patients with major depressive disorder. Biol Psychiatry. 2008;64(10):880-3.

54. Bifulco A, Bernazzani O, Moran PM, Jacobs C. The childhood experience of care and abuse questionnaire (CECA.Q): validation in a community series. $\mathrm{Br}$ J Clin Psychol. 2005;44(Pt 4):563-81.

55. Spijker J, De Graaf R, Bijl RV, Beekman AT, Ormel J, Nolen WA. Duration of major depressive episodes in the general population: results from The Netherlands Mental Health Survey and Incidence Study (NEMESIS). Br J Psychiatry. 2002;181:208-13.

56. Hassel S, McKinnon MC, Cusi AM, Macqueen GM. An Overview of Psychological and Neurobiological Mechanisms by which Early Negative Experiences Increase Risk of Mood Disorders. J Can Acad Child Adolesc Psychiatry. 2011;20(4):277-88.

57. Struber N, Struber D, Roth G. Impact of early adversity on glucocorticoid regulation and later mental disorders. Neurosci Biobehav Rev. 2014;38:17-37.

58. MacQueen G, Frodl T. The hippocampus in major depression: evidence for the convergence of the bench and bedside in psychiatric research? Mol Psychiatry. 2011;16(3):252-64.

59. Doom JR, Cicchetti D, Rogosch FA, Dackis MN. Child maltreatment and gender interactions as predictors of differential neuroendocrine profiles. Psychoneuroendocrinology. 2013;38(8):1442-54.

60. Young AS, Klap R, Sherbourne CD, Wells KB. The quality of care for depressive and anxiety disorders in the United States. Arch Gen Psychiatry. 2001;58(1):55-61.

\section{Submit your next manuscript to BioMed Central and we will help you at every step:}

- We accept pre-submission inquiries

- Our selector tool helps you to find the most relevant journal

- We provide round the clock customer support

- Convenient online submission

- Thorough peer review

- Inclusion in PubMed and all major indexing services

- Maximum visibility for your research

Submit your manuscript at www.biomedcentral.com/submit 